\title{
И. КИРЬЯНЕН
}

\section{К РАСЧЕТНЫМ МЕТОДАМ ПРИ ИСПОЛЬЗОВАНИИ ПРИНЦИПА МАКСИМУМА В ОПТИМИЗАЦИИ СЛОЖНЫХ ХИМИКО-ТЕХНОЛОГИЧЕСКИХ СИСТЕМ}

\section{(Представил О. Эйзен)}

При оптимизации сложных химико-технологических систем (СХTC) возникает необходимость в определении профилей технологических параметров в аппаратах. Для этого можно использовать методику принципа максимума. Утвержденная методика предусматривает для этой цели обобщенный критерий оптимизации - приведенные затраты всей СХTC. Однако применение такого критерия оптимума приводит к расчетным трудностям, в частности к невозможности аналитического нахождения производных. В настоящей работе предпринята попытка разрешить проблему численного дифференцирования по приблизительно рассчитанным данным.

Согласно методике технико-экономической оптимизации реакторов, находящихся в СXТC $\left[{ }^{1,2}\right]$, нужно найти зависимость

$$
\begin{gathered}
\Psi=\Psi(\tau) \approx \Psi^{\delta}(\tau), \\
\left\|\Psi(\tau)-\Psi^{\delta}(\tau)\right\| \leqslant \delta,
\end{gathered}
$$

где $\Psi$ - приведенные затраты всей СХТС на единицу продукта, $\tau-$ аргумент модели рассматриваемого аппарата, $\delta$ - величина, характеризующая ошибку. Величины $\Psi(\tau)$ обычно находятся расчетом CXTC при разных значениях $\tau$, в результате получаются величины $\Psi^{\delta}(\tau)$, которые имеют определенные ошибки.

В формуле (2) норму целесообразно взять в пространстве $L_{2}[a, b]$

$$
\|f\|_{L_{2}}^{2}=\int_{a}^{b}|f(x)|^{2} d x .
$$

В ходе определения профилей параметров нужно совместно решить системы дифференциальных уравнений [']:

$$
\begin{aligned}
& \frac{d x_{i}}{d \tau}=r_{i}(\bar{x}, \bar{u}) \quad(i=1,2, \ldots, m) \\
& \frac{\partial \lambda_{k}}{d \tau}=\frac{\partial^{2} \Psi}{\partial \tau \partial x_{k}}-\sum_{i=1}^{m} \lambda_{i}(\tau) \frac{\partial r_{i}\left(\bar{x}^{\prime}(\tau), \bar{u}(\tau)\right)}{\partial x_{k}} \quad(k=1,2, \ldots, m) \\
& \frac{d \lambda_{m+1}}{d \tau}=\frac{\partial^{2} \Psi}{\partial \tau^{2}}
\end{aligned}
$$

где $\bar{x}-$ состояние процесса $\left(\bar{x}^{\prime}=(\bar{x}, \tau)\right), \bar{u}-$ вектор управления, $\bar{\lambda}$ - вспомогательные функции. 
Қак видно, для решения системы (3) нужно рассчитать вторые производные $\frac{\partial^{2} \Psi}{\partial \tau \partial x_{k}}(k=1,2, \ldots, m)$ и $\frac{\partial^{2} \Psi}{\partial \tau^{2}}$ по приблизительным значениям $\Psi^{\delta}(\tau)$. В действительности же проблему представляет только расчет $\frac{\partial^{2} \Psi}{\partial \tau^{2}}$, так как

$$
\frac{\partial^{2} \Psi}{\partial \tau \partial x_{k}}=\frac{\partial^{2} \Psi}{\partial \tau^{2}} \cdot \frac{1}{r_{k}} \quad(k=1,2, \ldots, m) .
$$

Это т.н. некорректная линейная задача, поскольку разность

$$
\left\|\frac{d^{2}(\Psi(\tau))}{d \tau^{2}}-\frac{d^{2}\left(\Psi^{\delta}(\tau)\right)}{d \tau^{2}}\right\|
$$

может быть сколь угодно велика, в то время как разность

$$
\left\|\Psi(\tau)-\Psi^{\delta}(\tau)\right\|
$$

может быть сколь угодно мала. Следовательно, требуется специальный математический піодход - построение соответствующего регуляризатора [ $\left.{ }^{3}\right]$.

Предполагается, что функция $\Psi(\tau)$ определена на $[-1,1]$ и

$$
\Psi(0)=0, \quad \Psi^{\delta}(0)=0 .
$$

Если эти требования не выполняются, нужно провести линейное преобразование

$$
\begin{gathered}
\hat{\tau}=\frac{2}{b-a} \tau-\frac{b+a}{b-a}, \\
\hat{\Psi}^{\delta}(\widehat{\tau})=\Psi^{\delta}(\widehat{\tau})-\left.\Psi^{\delta}(\widehat{\tau})\right|_{\hat{\tau}=0},
\end{gathered}
$$

где $\hat{\tau}$ и $\widehat{\Psi}-$ новые переменные.

Так как прибавление произвольной постоянной не влияет на величину производной, то

$$
\frac{d^{2} \Psi}{d \hat{\tau}^{2}}=\frac{d^{2} \widehat{\Psi}}{\widehat{d \tau^{2}}}
$$

но замену аргумента нужно учитывать

$$
\frac{d^{2} \Psi}{d \tau^{2}}=\frac{d^{2} \Psi}{\widehat{d \tau^{2}}}\left(\frac{\hat{d \tau}}{d \tau}\right)^{2}=\frac{d^{2} \Psi}{\widehat{d \tau^{2}}} \cdot \frac{4}{(b-a)^{2}} .
$$

Функция $\widehat{\Psi}^{\delta}(\widehat{\tau})$ разлагается в ряд Фурье, В качестве ортонормированного базиса взяты полиномы Лежандра

$$
P_{n}(x)=\frac{1}{2^{n} n !} \cdot \frac{d^{n}\left[\left(x^{2}-1\right)^{n}\right]}{d x^{n}},
$$

которые имеют следующие рекуррентные связи:

$$
\begin{gathered}
(n+1) P_{n+1}(x)=(2 n+1) x P_{n}(x)-n P_{n-1}(x), \\
(n+1) \frac{d P_{n+1}(x)}{d x}= \\
=(2 n+1) P_{n}(x)+(2 n+1) x \frac{d P_{n}(x)}{d x}-n \frac{d P_{n-1}(x)}{d x} .
\end{gathered}
$$


Функция $\hat{\Psi}^{\delta}(\hat{\tau})$ заменяется полиномом $q_{n+1}(\hat{\tau})(n+1)$-й степени с тем, чтобы

$$
\begin{gathered}
\left\|\hat{\Psi}^{\delta}-q_{n+1}\right\| \leqslant \delta \\
\widehat{\Psi^{\delta}}(\widehat{\tau}) \sim \sum_{k=0}^{n+1} b_{k} P_{k}(\widehat{\tau}), \\
b_{k}=\frac{2 k+1}{2} \cdot \int_{-1}^{1} \widehat{\Psi^{\delta}}(\widehat{\tau}) P_{k}(\widehat{\tau}) \hat{d \tau}
\end{gathered}
$$

Тогда

$$
\left\|\widehat{\Psi}-q_{n+1}\right\| \leqslant 2 \delta
$$

В $\left[{ }^{4}\right]$ показано, как построить полином производной $p_{1}^{2 \delta}(\widehat{\tau})$, чтобы

$$
\lim _{\delta \rightarrow 0} p_{1}^{2 \delta}(\widehat{\tau})=\frac{d \widehat{\Psi}}{d \widehat{\tau}}
$$

и

$$
\left\|p_{1}^{2 \delta}(\widehat{\tau})-\frac{d \widehat{\Psi}}{d \hat{\tau}}\right\| \leqslant 2 \delta
$$

т. е. с той точностью, с которой известен полином $q_{n+1}(\hat{\tau})$, а именно

$$
p_{1}^{2 \delta}(\hat{\tau})=\sum_{k=1}^{n+1} \frac{b_{k}}{1+\alpha k^{2}(1+k)^{2}} \cdot \frac{d P_{k}(\hat{\tau})}{d \hat{\tau}}
$$

где $\alpha-$ положительное значение из условия

$$
\sum_{k=1}^{n+1} \frac{2}{2 k+1} h_{k}^{2} \frac{\alpha^{2} k^{4}(k+1)^{4}}{\left[1+\alpha k^{2}(k+1)^{2}\right]^{2}}=4 \delta^{2} .
$$

Степень полиномов (число $n+1)$ выбирается так, чтобы удовлетворялись условия

$$
\begin{gathered}
\int_{-1}^{1}\left(\hat{\Psi}^{\delta}\right)^{2} \hat{d \tau}-\sum_{k=0}^{n+1} \frac{2}{2 k+1} b_{k}^{2} \leqslant \delta^{2} \\
\sum_{k=0}^{n+1} \frac{2}{2 k+1} b_{k}^{2}>4 \delta^{2} .
\end{gathered}
$$

Для определения величины $\delta$ нужно знать абсолютную ошибку данных $\widehat{\Psi}^{\delta}(\widehat{\tau})$, т. е.

Тогда

$$
\left|\widehat{\Psi}^{\delta}(\widehat{\tau})-\widehat{\Psi}(\widehat{\tau})\right| \leqslant \Delta
$$

$$
\left\|\widehat{\Psi}^{\delta}-\widehat{\Psi}\right\|=\left(\left\|\widehat{\Psi^{\delta}}-\widehat{\Psi}\right\|^{2}\right)^{1 / 2}=\left(\int_{-1}^{1}\left|\widehat{\Psi^{\delta}}-\widehat{\Psi}\right|^{2} \widehat{d \tau}\right)^{1 / 2} \leqslant\left(2 \Delta^{2}\right)^{1 / 2}=\sqrt{2} \Delta
$$

T. e. $\delta=\sqrt{2} \Delta$. 
Зิначения характеристических величин при расчете вторых производных

\begin{tabular}{|c|c|c|c|c|c|c|c|c|}
\hline \multirow{2}{*}{$\begin{array}{c}\text { Про- } \\
\text { нзвод- } \\
\text { ная }\end{array}$} & \multirow{2}{*}{$\begin{array}{c}\delta \text { в фор- } \\
\text { муле } \\
(2)\end{array}$} & \multirow{2}{*}{$\begin{array}{c}n+1 \\
\text { в фор- } \\
\text { муле } \\
(8)\end{array}$} & $\alpha_{\text {нач }}$ & $\alpha_{\text {кон }}$ & \begin{tabular}{|c} 
число \\
ите- \\
раций
\end{tabular} & ||$I_{2 s}-\left.I_{s}\right|^{\text {max }}$ & $R_{2 s}^{\max }$ & $2 s$ \\
\hline & & & \multicolumn{3}{|c|}{ при решении формулы (13) } & \multicolumn{3}{|c|}{ в формуле (17) } \\
\hline
\end{tabular}

Первы й приме р

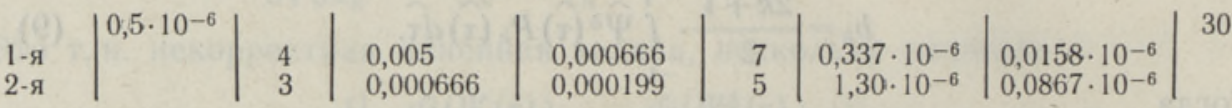

В торой п и м е

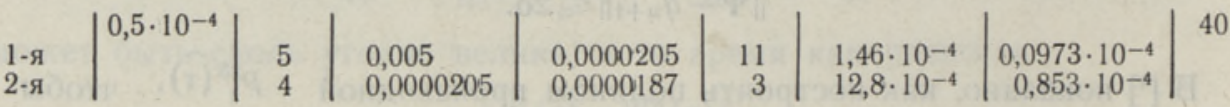

Интеграл в (9) целесообразно рассчитать по формуле Симпсона, где ошибку метода $R_{s}$ характеризует

$$
\left|R_{s}\right| \leqslant \frac{32}{180 \cdot s^{4}} \max _{\hat{\tau} \in[-1,1]}\left|\frac{d^{4}\left(\hat{\Psi}^{\delta}(\hat{\tau}) \cdot P_{k}(\widehat{\tau})\right)}{(\hat{d \tau})^{4}}\right|
$$

где $s$ - число используемых дискретных точек интегрируемой функции. Чтобы не потерять в точности, $s$ должен быть таким, чтобы

$$
\left|R_{s}\right| \leqslant \Delta \text {. }
$$

Когда поиск максимального значения четвертой производной по неравенству (16) затруднен, для определения значения $s$ можно использовать приближенную формулу

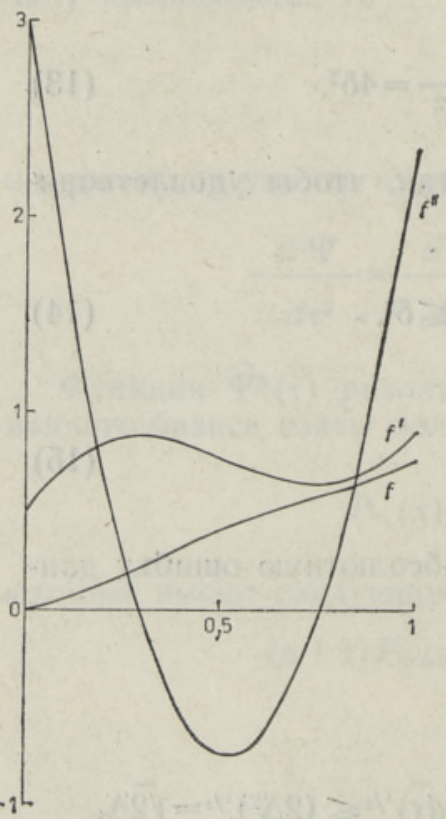

Рис. 1. Графики функции (18).

$$
\left|R_{2 s}\right| \approx \frac{1}{15}\left|I_{2 s}-I_{s}\right|,
$$

где $I_{2 s}$ и $I_{s}$ - значения рассчитываемых интегралов при разделении интервала интегрирования на $2 s$ и $s$ промежутков.

Переменную $\alpha$ из уравнения (13) можно определить методом Ньютона. Итерирование проводится до тех пор, пока точность $\boldsymbol{\alpha}$ не будет охарактеризована величиной $\Delta$. Начальное значение итерируемой переменной зависит от конкретного случая, но существование решения уравнения (13) гарантируется, причем область сходимости итераций обычно «большая».

Для нахождения второй производной описанную процедуру нужно повторить, соблюдая при этом выполнение требования (4) и делая в формулах (7)-(15) замены

$$
\begin{gathered}
p_{1}^{2 \delta} \rightarrow \Psi^{\delta}, \quad 2 \delta \rightarrow \delta, \quad \frac{d \widehat{\Psi}}{d \widehat{\tau}} \rightarrow \widehat{\Psi}, \\
p_{2}^{4 \delta} \rightarrow p_{1}^{2 \delta}, \quad \frac{d^{2} \widehat{\Psi}}{d \widehat{\tau^{2}}} \rightarrow \frac{d \widehat{\Psi}}{d \widehat{\tau}} .
\end{gathered}
$$




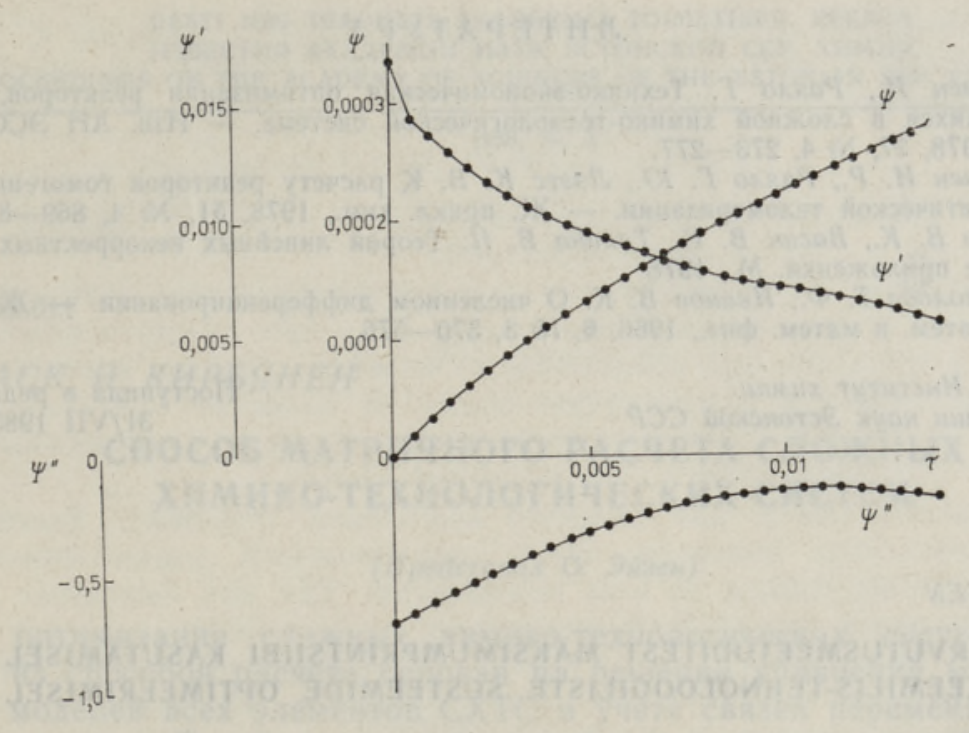

Рис. 2. Графики функции обратных значений приведенных затрат на единицу продукта и ее первой и второй производных.

$\begin{array}{lc}\text { Будем иметь } & \lim _{\delta \rightarrow 0} p_{2}^{4 \delta}(\widehat{\tau})=\frac{d^{2} \widehat{\Psi}}{\widehat{d \tau^{2}}} \\ \text { и } & \left\|p_{2}^{4 \delta}(\widehat{\tau})-\frac{d^{2} \widehat{\Psi}}{\widehat{d \tau^{2}}}\right\| \leqslant 4 \delta .\end{array}$

В общем, повторяя эту процедуру, можно найти производные сколь угодно высших порядков. Каждый раз, повышая порядок на единицу, мы вдвое теряем в точности.

Соответствующая программа составлена на языке ФОРТРАН. Для расчета значений полиномов Лежандра использованы рекуррентные связи (5) и (6).

Рассмотрим два примера. Во-первых, сложную функцию - комбинацию гиперболической функции с полиномом

$$
\begin{gathered}
f(x)=\text { th } x-(x-0,5)^{4}+0,625 \\
f^{\prime}(x)=\frac{4 \mathrm{e}^{-2 x}}{\left(1+\mathrm{e}^{-2 x}\right)^{2}}-4(x-0,5)^{3}, \\
f^{\prime \prime}(x)=\frac{8 \mathrm{e}^{-2 x}\left(\mathrm{e}^{-4 x}-1\right)}{\left(1+\mathrm{e}^{-2 x}\right)^{4}}-12(x-0,5)^{2}
\end{gathered}
$$

и, во-вторых, обратные значения приведенных затрат на единицу продукта конкретной СХТС - производства химических средств защиты растений. Судя по таблице и рис. 1,2 , метод обеспечивает получение нужных результатов за приемлемое машинное время.

В первом примере выбор именно такой функции не случаен. В рассматриваемом интервале (рис. 1) функция «почти» прямая, но вид ее производных довольно сложен. Таким образом, если недооценивать влияние ошибок в реальных случаях, т. е. если не элиминировать случайные отклонения, вызванные ошибками, то это может привести к изменению вида функций, а следовательно, и к неверным результатам. 
1. Кирьянен И., Раяло Г. Технико-экономическая оптимизация реакторов, находящихся в сложной химико-технологической системе. - Изв. АН ЭССР. Хим., 1978 , 27, № 4, 273-277.

2. Кирьянен И. Р., Раяло Г. Ю., Лээтс К. В. К расчету реакторов гомогенной каталитической теломеризации. - Ж. прикл. хим., 1978, 51, № 4, 869-874.

3. Иванов В. К., Васин В. В., Танана В. П. Теория линейных некорректных задач и ее приложения. М., 1978.

4. Долгополова T. Ф., Иванов В. К. О численном дифференцировании. - Ж. вычисл. матем. и матем. физ., 1966, 6, № $3,570-576$.

Институт химии

Академии наук Эстонской ССР
Поступила в редакщию 31/VII 1985

\section{KIRJANEN}

\section{ARVUTUSMEETODITEST MAKSIMUMPRINTSIIBI KASUTAMISEL KEEMILIS-TEHNOLOOGILISTE SUSTEEMIDE OPTIMEERIMISEL}

Tehnoloogiliste parameetrite optimaalsete profiilide leidmiseks aparaatides võib kasutada maksimumprintsiibi metoodikat. Vaadeldes aparaati keemilis-tehnoloogilise süsteemi elemendina ja kasutades üldistatud optimeerimiskriteeriumi, tekivad arvutusraskused. Oheks probleemiks on üldistatud optimeerimiskriteeriumi teiste tuletiste arvutamine vaadeldava aparaadi argumendi järgi. Artiklis vaadeldakse võimaiust selle probleemi lahendamiseks. Väljatöötatud metoodika on kasutatav palju üldisemalt.

\section{KIRJAINEN}

\section{ABOUT THE COMPUTATIONAL TECHNIQUES OF USING MAXIMUM PRINCIPLE IN OPTIMIZATION OF A CHEMICAL ENGINEERING SYSTEM}

In the calculation of the optimal profile of technological parameters, the maximum principle can be used. Computational difficulties will crop up if we consider the examining apparatus an element of a chemical engineering system, and if the generalized optimum criterion is applied. A problem is the calculation of the second derivative of the generalized optimum criterion with respect to the argument of the examining apparatus. The author proposes a possibility to solve that problem. The method elaborated can be applied on a wider scale as well. 